\title{
Développement d'une source plasma-laser pour la lithographie dans l'extrême ultraviolet
}

\author{
G. Soullié, C. Lafon, R. Rosch, D. Babonneau, F. Garaude, S. Huelvan, \\ T. Trublet, L. Bonnet et R. Marmoret
}

CEA/DAM lle de France, BP. 12, 91680 Bruyères-le-Châtel, France

\begin{abstract}
Résumé. Cet article présente notre rôle au sein du projet PREUVE (PRogramme Extrême UV), en particulier, le développement et la caractérisation d'une source EUV plasma-laser. Cette source est basée sur l'utilisation du rayonnement EUV émis en face arrière d'une cible irradiée par laser. Le laser utilisé possède les caractéristiques suivantes : énergie de $2 \mathrm{~J}$, durée d'impulsion de $7 \mathrm{~ns}$ et taux de répétition de $10 \mathrm{~Hz}$. La cible est composée d'étain supporté par un film de mylar. Nous présentons ici les diagnostics développés pour la caractérisation de cette source ainsi que les derniers résultats obtenus.
\end{abstract}

\section{INTRODUCTION}

Actuellement, la lithographie qui permet la réalisation de circuits de semi-conducteurs (mémoires et processeurs d'ordinateurs par exemple) utilise l'ultraviolet à $193 \mathrm{~nm}$ et bientôt à $157 \mathrm{~nm}$. Pour cela, le rayonnement UV passe au travers d'un masque sur lequel est reproduit le motif à graver dans une plaque de silicium, puis insole la résine déposée sur cette plaque de silicium. Entre le masque et le silicium, des optiques en transmission sont utilisées pour réduire les motifs du masque. La résine est ensuite révélée par un bain chimique pour faire apparaître sur le silicium les motifs réduits du masque. Pour réaliser des gravures de taille plus petite, il est nécessaire de diminuer la longueur d'onde pour abaisser la limite de diffraction. Ainsi, pour réaliser des circuits d'une largeur inférieure à $50 \mathrm{~nm}$, une nouvelle technique de lithographie est nécessaire. Une solution prometteuse est la lithographie dans l'extrême ultraviolet (EUVL) ( $\lambda=13,5 \mathrm{~nm}$ ) [1]. Comme le rayonnement EUV est très fortement absorbé par tous les matériaux, y compris l'air, il n'est plus possible d'employer des optiques en transmission, il faut maintenant travailler sous vide et avec des optiques en réflexion. En 1997, deux programmes pour développer cette technique ont été mis en place par des industriels américains et japonais des semi-conducteurs. En France, un programme (PREUVE) financé par le Ministère de l'Économie, des Finances et de l'Industrie regroupant des industriels et des laboratoires du CNRS et du CEA, a été réalisé entre novembre 1999 et août 2002. Dans PREUVE, le CEA/DAM a été impliqué dans le développement d'une source, la métrologie des optiques et l'intégration du Banc d'Essai pour la Lithographie (BEL). 


\section{LA SOURCE EUV}

Pour cette source, on utilise le rayonnement émis en face arrière d'une cible solide plane irradiée par un laser à éclairement moyen $\left(<10^{13} \mathrm{~W} / \mathrm{cm}^{2}\right)$. Les originalités de notre solution sont la directivité du rayonnement EUV (émission dans un cône de $60^{\circ}$ ) qui permet une collection plus efficace et la faible quantité de débris produits. En effet, ces derniers projetés sur les optiques abaissent considérablement leur durée de vie. Comme l'absorption du rayonnement à $13.5 \mathrm{~nm}$ est très importante, un autre intérêt est que le rayonnement EUV émis ne retraverse pas de matière, comme dans le cas des jets d'agrégats. Le principal inconvénient est la destruction de la cible après chaque tir. Nous verrons par la suite la solution que nous avons adoptée pour résoudre ce problème.

La modélisation numérique à l'aide de codes $\mathrm{FCI}$ développés au CEA/DAM a permis d'optimiser les paramètres du laser (flux, durée, longueur d'onde) et de la cible (composition, épaisseur).

Les premiers calculs, à paramètres laser donnés, montre que l'étain est le corps le plus émissif dans le domaine 90-100 eV. Ces simulations sont en accord avec les résultats obtenus par Spitzer et ses collaborateurs [2] pour le rayonnement émis en face avant d'une cible solide. Des cibles composites ont été aussi étudiées pour augmenter le rendement de conversion X.

Pour toutes ces configurations de cibles, de nouveaux calculs ont été effectués pour optimiser les paramètres du laser. Nous en avons déduit les caractéristiques suivantes : une durée d'impulsion de $5-10 \mathrm{~ns}$, une longueur d'onde de $1.06 \mu \mathrm{m}$ et un éclairement compris entre $10^{10}-10^{13} \mathrm{~W} / \mathrm{cm}^{2}$. Une première campagne de mesures préliminaires a été réalisée courant 2000 au Centre Laser Franco-Allemand (CLFA - Fort d'Arcueil, France) en utilisant un laser ayant les caractéristiques suivantes : $30 \mathrm{~J}, 10 \mathrm{~ns}$ et $1.06 \mu \mathrm{m}(\omega)$. Malheureusement, l'impulsion de ce laser était trop éloignée d'une impulsion de forme carrée et ainsi le rendement de conversion $\mathrm{X}$ était très faible. Par contre, les résultats ont montré la bonne directivité du rayonnement émis : un rapport 6 entre les signaux de deux détecteurs a été obtenu, l'un était placé à $15^{\circ}$ et l'autre à $45^{\circ}$.

Nous avons donc acheté un laser Nd:YAG de la société THALES avec les caractéristiques suivantes : $2 \mathrm{~J}, 7 \mathrm{~ns}$ et $1,06 \mu \mathrm{m}(\omega)$. Ce laser peut fonctionner soit en monocoup ou en cadencé jusqu'à $10 \mathrm{~Hz}$. La stabilité de l'intensité laser tir à tir est inférieure à $3 \%$. Nous avons mesuré en mode injecté une impulsion de $7,2 \mathrm{~ns}( \pm 2 \%)$ avec une énergie laser de $1,8 \mathrm{~J}$.

Nous nous sommes aussi équipés d'un chambre d'interaction ainsi que des diagnostics pour caractériser la source créée (figure 1). Pour un fonctionnement monocoup, les cibles sont disposées suivant une spirale sur un disque. Un mouvement combinant une translation et une rotation permet de mettre en position la cible au foyer du faisceau laser. Dans son fonctionnement à $10 \mathrm{~Hz}$, le disque contiendra 1700 cibles.

Pour la caractérisation de cette source, nous avons développé un ensemble de diagnostics pour faire l'image du point source et mesurer le flux émis (Figure 1).

Pour mesurer le flux émis en différents points du spectre, six voies de mesure sont disposées en couronne. Elles sont composées chacune d'un collimateur et d'un goniomètre. Sur ce dernier, on peut installer un miroir interférentiel multicouche ainsi qu'un détecteur muni d'un filtre.

Par exemple, pour une mesure à $13,5 \mathrm{~nm}$, nous utilisons un filtre de zirconium de $0,35 \mu \mathrm{m}$ d'épaisseur qui élimine le visible et l'UV proche. La multicouche possède une bande passante de $3 \mathrm{eV}$ à $92 \mathrm{eV}(13,5 \mathrm{~nm})$.

La métrologie en absolu des éléments d'une voie multicouche a été réalisée sur la ligne SA23 de l'anneau synchrotron SUPER-ACO [3]. La précision spectrale de cette ligne est de 0,1 eV. Nous 
avons donc mesuré le pouvoir réflecteur des multicouches ainsi que la transmission des filtres de zirconium. Nous avons ainsi pu calculer les caractéristiques de ces différents éléments par un fit. La précision sur la mesure du pouvoir réflecteur de la multicouche est de $3 \%$.

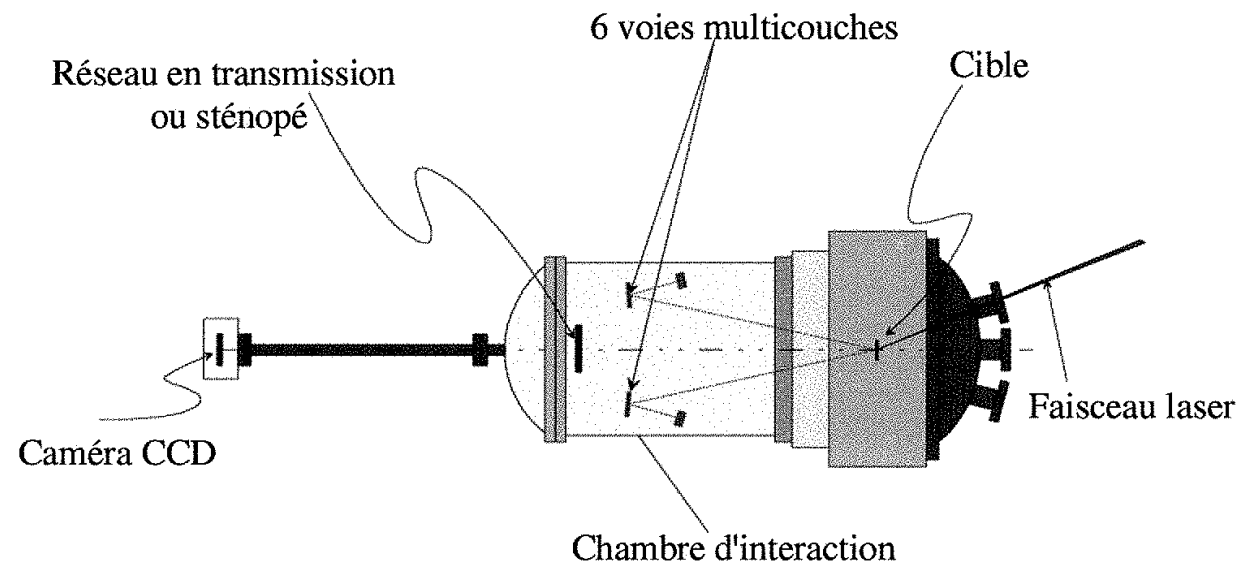

Figure 1. Les diagnostics de la source EUV
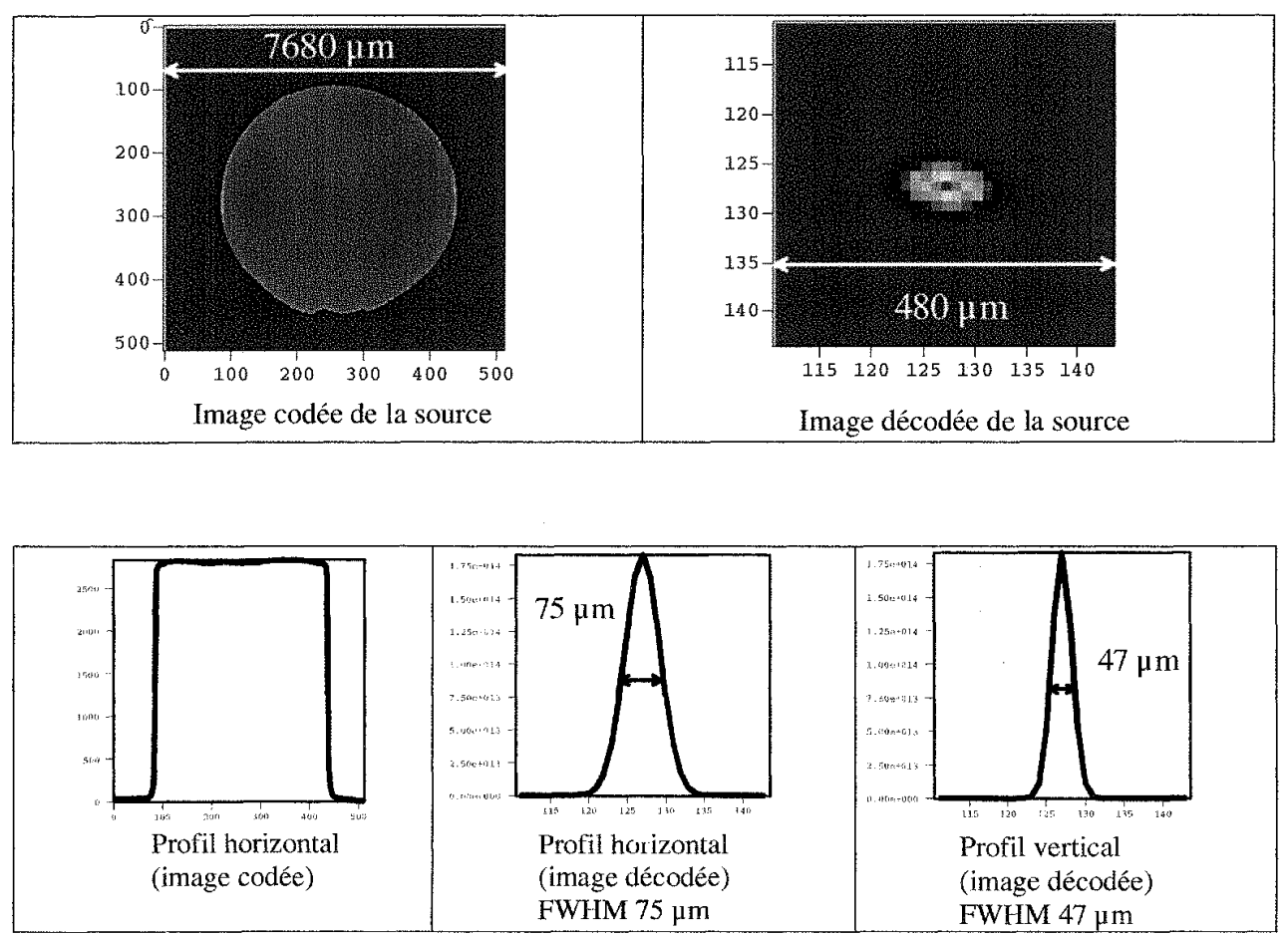

Figure 2. Image du point source EUV 
Pour réaliser l'image du point source EUV, nous avons utilisé la technique de l'imagerie de pénombre [4]. Nous avons utilisé un sténopé de diamètre $3 \mathrm{~mm}$ dont nous avons fait l'image sur une caméra CCD éclairée en face arrière. Pour atténuer le visible et découper une bande dans le spectre émis, nous avons placé un filtre de zirconium devant le sténopé.

Comme le faisceau laser arrive sur la cible avec un angle de $15^{\circ}$, on retrouve bien, sur l'image décodée, une forme de source elliptique (Figure 2). On remarque nettement que la résolution est limitée par la taille des pixels de la caméra $(20 \mu \mathrm{m})$. Une nouvelle expérience est prévue en changeant la taille du sténopé et le grandissement afin d'améliorer la résolution de l'image.

Comme décrit par la théorie, il existe une épaisseur de cible nominale pour un couple éclairement laser-matériau. Nous avons vu que l'étain était le matériau le plus émissif autour de $13.5 \mathrm{~nm}$. Sur la figure 3, nous montrons la variation de l'énergie émise suivant l'épaisseur d'étain. On note la présence d'un maximum pour une épaisseur voisine de $0,35 \mu \mathrm{m}$

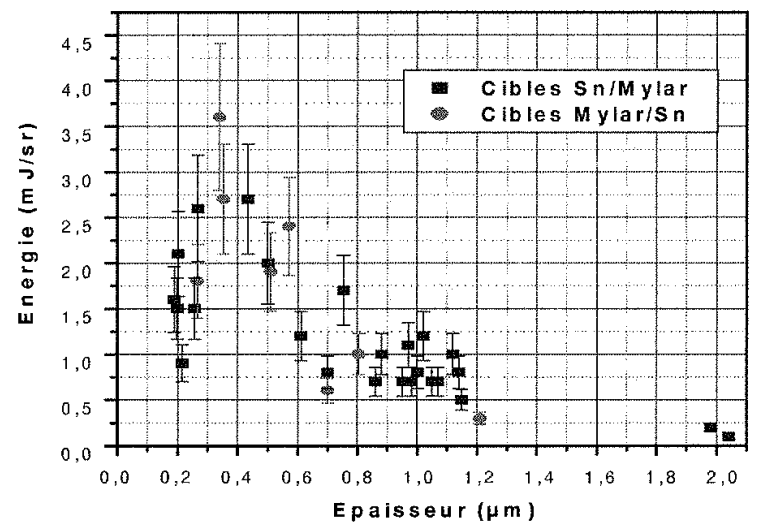

Figure 3. Variation de l'énergie EUV émise suivant l'épaisseur de la cible

Grâce à la voie multicouche centrée sur $92 \mathrm{eV}$, on mesure une énergie moyenne émise à $92 \mathrm{eV}$ $(13.5 \mathrm{~nm}$ ) par impulsion laser est de $3 \mathrm{~mJ} / \mathrm{sr}$ dans une bande de $3 \mathrm{eV}$ autour de $92 \mathrm{eV}$. Nous avons ainsi une efficacité de conversion en face arrière de $0.2 \%$.

\section{CONCLUSION ET PERSPECTIVES}

Nous avons développé une source EUV pour la lithographie sur le principe de l'émission en face arrière d'une cible mince éclairé par un laser d'intensité modéré. Les premiers résultats nous ont montré la bonne stabilité spatiale et temporelle du faisceau laser permettant une bonne reproductibilité de la tache de focalisation. L'énergie moyenne à $13,5 \mathrm{~nm}$ par impulsion laser est de $3 \mathrm{~mJ} / \mathrm{sr}$ dans une bande de $3 \mathrm{eV}$ à $92 \mathrm{eV}$. La stabilité de l'intensité émise tir à tir est de $15 \%$. Dans le futur proche, nous allons continuer la caractérisation de cette source et ainsi :

améliorer la résolution de l'imagerie de pénombre (sténopé de $1 \mathrm{~mm}$ ),

caractériser le cône d'émission par la mise en place d'un goniomètre dans la chambre d'interaction,

caractériser l'émission des débris,

et mettre au point le fonctionnement de la source à $10 \mathrm{~Hz}$.

\section{Remerciements}

Nous remercions A. Rouyer pour son aide sur le dimensionnement et le dépouillement des images de pénombre. 


\section{Références}

[1] C. W. Gwyn "Extreme Ultaviolet Lithography - White paper", Nov. 1999.

[2] R. C. Spitzer and al., "X-Ray Production from Laser Produced Plasmas from Soft X-Ray Projection Lithography Applications", in Conference on Lasers and Electro-optics, Baltimore MD, May 2-7, 1993.

[3] Ph. Troussel, C. Bobin, C. Rémond, Ph. Stemmler et B.Villette, "Métrologie autour d'installations plasma-laser. Utilisation du rayonnement synchrotron", J. Phys. IV France 11 (2001) 179-182.

[4] A. Rouyer, "A new simple method for decoding penumbra images: the filtered autocorrelation", R.S.I., à paraître. 